\title{
An Approach to Fault Diagnosis Considering False Alarm and Middle State
}

\author{
Xiangqian Li \\ School of Mechanical Engineering \\ Beijing Institute of Technology \\ Beijing, China \\ e-mail: lxqljy@163.com
}

\author{
Shikai Jing \\ School of Mechanical Engineering \\ Beijing Institute of Technology \\ Beijing, China \\ e-mail: jingshikai@bit.edu.cn
}

\author{
Jingtao Zhou \\ School of Mechanical Engineering \\ Northwestern Polytechnical \\ University \\ Xi’an, China \\ e-mail: jtzhou.office@gmail.com
}

\begin{abstract}
In order to monitor the false alarm and fault middle state in the process of fault diagnosis simultaneously, a mixed Hidden Markov Model (HMM) considering the false alarm and fault middle state was proposed to reduce the false alarm and monitor the middle state of the system. First of all, the state classification in the process of the complex system state monitoring was studied. The system states were divided into normal state, intermittent failure, random failure, intermediate state and failure state, and then the mixed HMM was established to reflect the true state of the system. Secondly, the effective feature vectors were extracted based on wavelet packet decomposition method to eliminate redundancy and high dimension of original characteristic signal. Then all kinds of the HMM states were obtained through the model training, and fault diagnosis was implemented through inputting feature vector. At last, the feasibility and effectiveness of the method was verified by an example.
\end{abstract}

Keywords-wavelet packet decomposition; HMM; analog circuit; fault diagnosis

\section{INTRODUCTION}

Fault diagnosis has been an extensive research and achieved fruitful results since the 1970s in domestic and overseas. However, with the development of technology, the increasing of complexity and integration of all kinds of system has brought great challenge to monitoring and maintenance of system. According to the different of fault characteristics and the duration, fault conditions of system was divide by pulse changing fault, abrupt changing fault and gradual changing fault [1]. (1) The pulse changing fault, such as the intermittent fault and transient fault appears in the system, that usually lasts shorter time was mainly caused by the defects of system internal or specific external environment. (2) The abrupt changing fault often occurs without warning that duration is usually permanent and only to identify and diagnose after failure. (3) The gradual changing fault was occurred slowly, which has a gradually changing tend and usually because system component parameters deteriorate caused by vibration and fever of the system in the process of long operation.

The pulse changing fault was monitored effectively that can reduce fault false alarm rate and improve the diagnostic performance of the system. The abrupt changing fault was difficult to early identification and prevention due to the lack of signs. The early fault of system can be detected by identification of gradual changing fault, and to be prevented immediately through take effective measures. However, the traditional fault diagnosis technology has been difficult to meet the demand of system maintenance [2-3] due to the many factors of system arise from false alarm and difficult to identify the early fault features. For example, the traditional fault diagnosis is usually set the appropriate threshold as determine the basis of the fault. This condition monitoring method based on the threshold lack flexibility and adaptability, because it is difficult to determine the threshold and need to set more monitoring point in the system. The biggest shortcoming of the traditional fault diagnosis is that the system state is only divided into two states of normal and fault. So the traditional fault diagnosis cannot eliminate the failure alarm which causes from the intermittent and random faults, and cannot monitor intermediate state for system difficult to monitor the gradual changing fault.

Consequently, the premise and guarantee of intelligent fault diagnosis is to establish a model to reflect the true state of system based on different fault types to extract fault feature accurately and effectively. Wavelet transform is a feature extraction method for more research in the fault diagnosis field. Wavelet packet decomposition classified arbitrary signal include sinusoidal signal into the corresponding frequency band, and can effective extract the characteristics of signal [4-5]. HMM reflect to the internal state through observed the observation values of sequence. HMM has self-learning and self-adaptive ability and very suitable for pattern recognition and fault monitoring to complex systems. HMM has been widely used in field of speech recognition and fault diagnosis [6-8]. [9-10] built the HMM of electronic systems, which divided the states of electronic system into normal, weak fault state, intermediate fault state and fault state. The model is used to response of health process of electronic system, predict the running state of the system and realize the early warning of fault. An intelligent BIT fault diagnosis method based on Hidden Markov model was presented in literature [1], the transient and intermittent faults as the intermediate state of the system were identifiable separately in order to reduce the false alarm rate of system.

Based on the above research results, a mixture HMM was established in the paper combing with the advantage of wavelet packet analysis and HMM and the fault types of system. The HMM models of the system state were 
established through extracting the feature of original signal by wavelet packet, which used to monitor intermittent faults and the gradual changing fault of system at the same time.

\section{THE MiXTURE HMM}

\section{A. The theory of HMM}

The HMM is different from Markov chains. Because of the actual state of the model is implicit and cannot be observed directly, so it is called HMM. HMM is a probabilistic model for describing the statistical characteristics of random process that consisting of two random processes. One of which is describe the transfer of state, another describe the statistical relationship between state and observation value. The HMM was described usually with five elements $\lambda=(N, M, \pi, A, B)$ or recorded as $\lambda=(\pi, A, B)$, where $\mathrm{N}$ is the number of the states in the model, $M$ represents the number of distinct observation symbols per state. The other parameters are defined as follow.

(1) $\pi$, the initial state probability distribution. $\pi=\left(\pi_{1}, \pi_{2}, \ldots, \pi_{N}\right)$, where

$$
\pi_{i}=p\left(X_{i}=S_{i}\right), 1 \leq i \leq N .
$$

(2) A, the state transition probability $\begin{aligned} \text { matrix. } A & =\left\{a_{i j}\right\}_{N \times N} \text {, where } \\ & a_{i j}=P\left(X_{i+1}=S_{j} \mid x_{i}=S_{i}\right), 1 \leq i, j \leq N .\end{aligned}$

(3) $B$, the observation symbol probability matrix. $B=\left\{b_{j k}\right\}_{N \times M}$, where

$$
b_{j k}=P\left(y_{i}=v_{k} \mid x_{i}=S_{j}\right), 1 \leq j \leq N, 1 \leq k \leq M .
$$

Because A is used to describe the transition probability of each state in the model, so the structure of HMM model can be described by A. The HMM structure is divided into ergodic type and left-right type [10]. Ergodic structure refers to each state in the system can be transferred to any other state. So, each element of a matrix in the ergodic structure cannot be zero. The left-right structure defined the state in the system is sequential transfer and irreversible. In other words, the state of current state can only be transferred to the serial number higher or equal to the current state in accordance with sequence, and the initial probability $\pi$ must satisfy the following equation.

$$
\pi_{i}= \begin{cases}1 & i=1 \\ 0 & i>1\end{cases}
$$

\section{B. The mixture HMM}

At present, most of the researches on fault diagnosis based on HMM are establish the left-right model. These models assumes that the system state is the continuity and irreversible. However, a single left-right HMM cannot fully describe the system state because of the transient and intermittent faults were existed in the process of actual operation. Therefore, a hybrid HMM structure was constructed based on the left-right structure in this paper, including all kinds of the running condition of the system to reflect the true state of the system. The hybrid HMM divided the state of system into normal state, intermittent fault, random fault, intermediate state and failure state. As shown in Fig.1, the mixed HMM was established according to the mutual transformation relationship and performance of the system state.

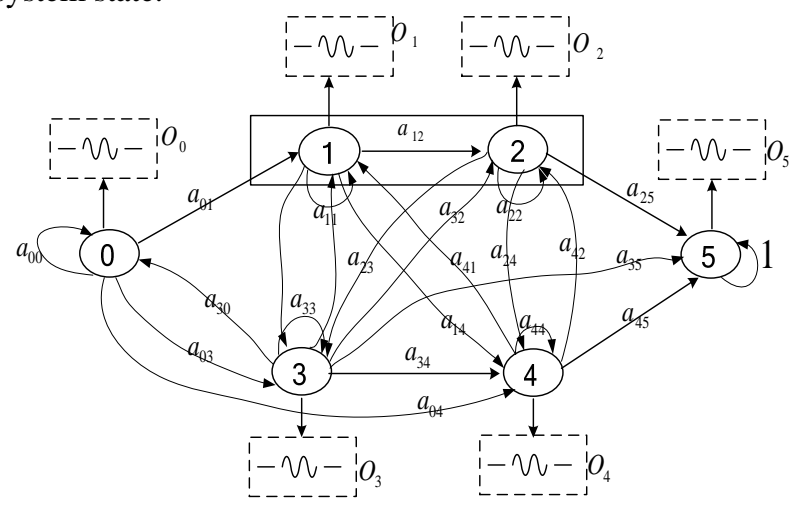

Figure 1. The mixture HMM

Where 0 indicates normal state, 1 and 2 are the intermediate state ( 1 is the slight deterioration in early and 2 is the serious deterioration in middle), 3 is the transient fault, 4 is intermittent fault, and 5 is the permanent fault. $a_{i j}(\mathrm{i}, \mathrm{j}=0$, $1,2,3,4,5)$ is the transfer probability between the state. $O_{i}$ ( $\mathrm{i}=0,1,2,3,4,5)$ is the observation value. Transient fault and intermittent faults are temporary failure mostly caused by environmental factors, and the conversion of between the normal state and the intermediate state is reversible. Due to the performance of transient fault and intermittent fault is similar to the effect on the system, the state of 3 and 4 are referred to intermittent fault for convenience [3]. The mixture HMM with five states consider the intermittent fault and gradual changing fault, and take them as the independent state of the system monitoring compared to diagnosis method of the normal and fault state.

Usually, the initial state of the system is always normal; the initial probability of the states $\pi$ is set as $\pi=\left[\begin{array}{llll}1, & 0,0,0,0\end{array}\right]$.

The state transition probability matrix A can reflect the correlation information of process state sequence for some modeling problem of related to sequences. The A matrix for mixture HMM is given below:

$$
A=\left[\begin{array}{lccccc}
a_{00} & a_{01} & 0 & a_{03} & a_{04} & 0 \\
0 & a_{11} & a_{12} & a_{13} & a_{14} & 0 \\
0 & 0 & a_{22} & a_{23} & a_{24} & a_{25} \\
a_{30} & a_{31} & a_{32} & a_{33} & a_{34} & a_{35} \\
0 & a_{41} & a_{42} & a_{43} & a_{44} & a_{45} \\
0 & 0 & 0 & 0 & 0 & 0
\end{array}\right]
$$

The HMM is divided into two categories, namely, continuous HMM (CHMM) and discrete HMM (DHMM), 
which are discerned by the observed signal. For CHMM the observation is continuous, the initial value of the observation probability matrix $\mathrm{B}$ can be obtained by the $\mathrm{K}$ mean algorithm [12]. For DHMM the observation is discrete, the properties of observed value corresponding to a state are represented by a set of probability.

There are three basic algorithms in HMM need to solve $[1,13]$. (1) Calculation probability, the $p(O \mid \lambda)$ was calculated usually by the forward-backward algorithm for any initial model $\lambda$ and observation sequence $O$. (2) The iterative convergence, initial model and observation sequence were fitted better by adjusting the model parameters and iterative to model convergence. The EM and Baum-Welch are common algorithms. (3) The state estimation, the optimal state sequence corresponds to the observation sequence was calculated by Viterbi algorithm.

\section{Fault Monitoring Process Of Mixture HMM}

The procedure for the fault diagnosis can be described as follows:

(1) Wavelet packet decomposition. Firstly, the training sample data was processed by $\mathrm{N}$ layer wavelet packet decomposition and the wavelet packet are reconstructed to obtain the signal energy of each sub-band.

(2) Extract fault feature. The fault features are extracted by data pretreatment, fault feature vector were constructed through the normalized processing.

(3) HMM training. For each state type, select any $\mathrm{K}$ feature vector to compose of a set of observations, which used to train the HMM, state type for the hidden state, each state type training a HMM model.

(4) Pattern recognition. The testing sequence is sent to HMM models after feature extraction, and the corresponding probabilities are calculated, and the most probable system condition is considered as the one that has the maximal value of the probability.

\section{EXAMPLE VERIFICATION}

\section{A. Simulation modeling and feature extraction}

Fig. 2 shows a biquad high-pass filter with components set to their nominal values resulting in a cut-off frequency of $10 \mathrm{kHz}$ [11]. The nominal values of the components were indicated in Fig.2. We introduce faulty components in the circuit and vary resistors and capacitors within the standard tolerances of $\pm 5 \%$ and $\pm 10 \%$, respectively. The R1, R2, R3, R4, C1 and C2 were set fault element. For each of the fault component, two soft-fault classes can be shown as follows: a class for the component values larger than the nominal one (labeled by $\uparrow$ ) and the other for the component values smaller than the nominal one (labeled by $\downarrow$ ).

The failure mode of $\mathrm{R}$ is beyond $\mathrm{R} \pm 50 \%$, failure mode of $\mathrm{C}$ was beyond $\mathrm{C} \pm 50 \%$. Take the increase process of $\mathrm{C} 1$ as an example, in order to monitor the middle fault, which will be divided into early and mild deterioration $C 1 \in(1.10 C, 1.25 C]$, serious deterioration of the middle $C 1 \in(1.25 C, 1.50 C]$ and complete fault $C 1 \in(1.50 C, 3.0 C]$. This was used to simulate the failure process of the circuit. In order to simulate intermittent fault of the system, add a diode at the power source to get the intermittent signal. Hence, 15 fault classes are simulated and output of the filter is used as the test node.

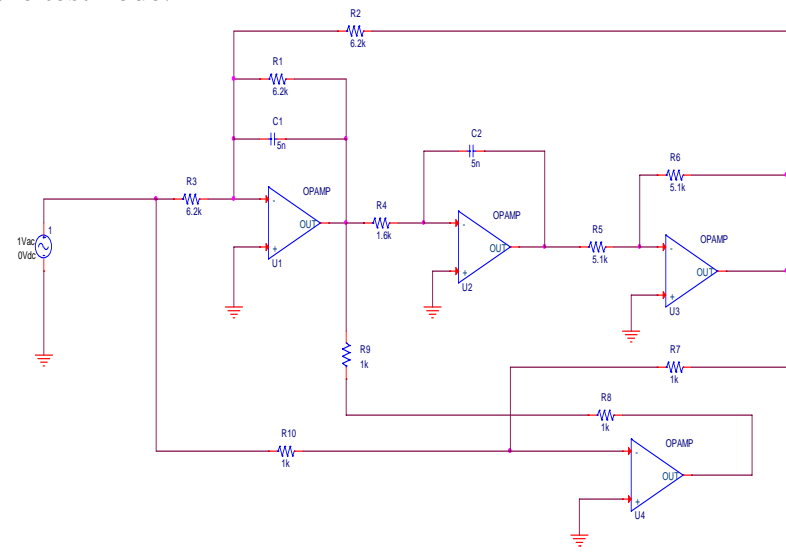

Figure 2. Four op-amp biquad high-pass filter

The amplitude and the angle of the circuit (VAC) were set to $1 \mathrm{~V}$ and 0 . Take the output of the circuit as the measuring point. The circuit states were communicated analysis respectively by OrCAD PSPICE software, the frequency range is $1 \mathrm{KHZ}-100 \mathrm{KHZ}$. The circuit fault feature was extracted through Monte Carlo simulation. In our work, only single incipient fault has been considered. For 15 states, each state was simulated respectively 50 times, a total of 750 samples, which are divided into 450 training data and 300 testing data. All the sample data were analyzed by three layer wavelet packet based on wavelet packet analysis technology, and construct to the feature vector and normalized. Because of the influence of element normal tolerance and the early fault is difficult to identify, the sym5 wavelet was chosen through a lot of experiments.

A part sample data after wavelet packet decomposition and normalized are shown in Table I.

TABLE I. PART DATA SAMPLES OF THE NORMALIZED

\begin{tabular}{|c|c|c|c|c|c|c|c|c|}
\hline failure classes & E3O & E3I & E32 & E33 & E34 & E35 & $E 36$ & E37 \\
\hline normal & 0.9678 & 0.1684 & 0.0415 & 0.1722 & 0.0119 & 0.0267 & 0.0457 & 0.0255 \\
\hline $\mathrm{R} 14$ & 0.9349 & 0.2016 & 0.0743 & 0.2486 & 0.0397 & 0.0648 & 0.0989 & 0.0485 \\
\hline RIt & 0.9948 & 0.0827 & 0.0093 & 0.0585 & 0.0008 & 0.0043 & 0.0042 & 0.0051 \\
\hline Early fault & 0.9677 & 0.1213 & 0.0414 & 0.1989 & 0.0090 & 0.0313 & 0.0702 & 0.0408 \\
\hline $\begin{array}{c}\mathrm{Cl} \\
\text { Middle fault }\end{array}$ & 0.9672 & 0.1060 & 0.0787 & 0.2023 & 0.0101 & 0.0131 & 0.0635 & 0.0439 \\
\hline $\begin{array}{c}\mathrm{Cl} \\
\text { complete fault }\end{array}$ & 0.9638 & 0.1278 & 0.1069 & 0.1979 & 0.0212 & 0.0059 & 0.0481 & 0.0378 \\
\hline $\mathrm{R} 2 \uparrow$ & 0.9824 & 0.1243 & 0.0537 & 0.1266 & 0.0043 & 0.0026 & 0.0204 & 0.0098 \\
\hline $\mathrm{R}_{2} \downarrow$ & 0.9502 & 0.2639 & 0.0575 & 0.1112 & 0.0145 & 0.0155 & 0.0954 & 0.0480 \\
\hline $\mathrm{R} 3 \uparrow$ & 0.9178 & 0.1061 & 0.0332 & 0.1934 & 0.0122 & 0.0305 & 0.0611 & 0.0317 \\
\hline${ }^{R} 3 \downarrow$ & 0.9669 & 0.2145 & 0.0448 & 0.0125 & 0.0078 & 0.0172 & 0.0253 & 0.0163 \\
\hline $\mathrm{R} 4 \uparrow$ & 0.9859 & 0.0731 & 0.0421 & 0.1395 & 0.0022 & 0.0086 & 0.0310 & 0.0186 \\
\hline${ }^{R} 4 \downarrow$ & 0.9092 & 0.3759 & 0.0753 & 0.0806 & 0.0248 & 0.0203 & 0.1191 & 0.0676 \\
\hline intermittent faul & 0.9358 & 0.3455 & 0.0421 & 0.0551 & 0.0033 & 0.0045 & 0.0081 & 0.0067 \\
\hline
\end{tabular}

\section{B. HMM modeling and fault recognition}

The HMM model of system is established. $\lambda_{1}, \lambda_{2}, \ldots$, $\lambda_{15}$ are used to express each fault state of circuit. At the initial moment, the system is in a normal state,

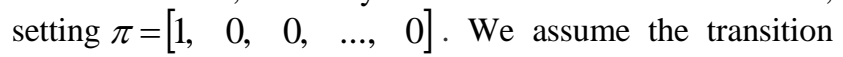


probability from one state to another state is same, and set the value 0.5. But the state is not reversible in the decay process.

The probability matrix of initial observation is accessed random in MATLAB. The Baum-Welch algorithm is used to train the 450 samples data. The feature vectors are conducted scalar quantization using Lloyd algorithm before training [13]. 15 HMM recognition models corresponding to the 15 circuit state are obtained after the end of the training.

After the HMM training completed, the remainder of the 300 test samples were input to state HMM model for testing. The scalar quantization coding is done for the feature vector the same of using Lloyd algorithm before testing [13]. 10 experiments are conducted for each states and the averages are used because of the initial parameters of the HMM with a certain randomness degree. As the results shown in Table II, the intermittent faults and the gradient fault were diagnosed successfully and higher recognition rate by the proposed method.

TABLE II. The StATE RECOGNITION RATE

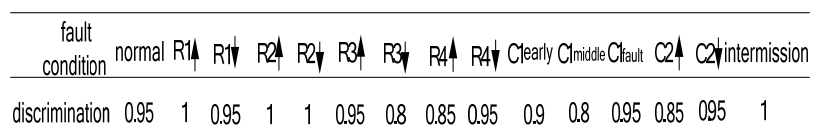

\section{CONCLUSION}

In this paper, a compound Hidden Markov Model for the false alarm and the intermediate statuses of artificial circuit is proposed. The model has taken the feature and advantage of both wavelet packet decomposition and HMM to achieve artificial circuit's fault diagnosis. Taking four op-amp biquad high-pass filter as an example, the effectiveness of wavelet packet in extracting fault information has been proved. The compound HMM model could reflect the system states veritably, and the effectiveness of diagnosing both early faults and intermittent faults are also proved. However, since there are too many system states, the diagnosis takes a long time to operate. The further research will focus on improving the real time of diagnosis and health management method for system.

\section{ACKNOWLEDGMENT}

The authors would like to thank the reviewers for their detailed revises and constructive comments. This work was supported by the International Cooperation Ministry of Science and Major Project (NO. 2011DFB10090), the National Natural Science Foundation of China through approval (NO. 61104169), and Specialized Research Fund for the Doctoral Program of Higher Education through approval of China (NO. 2010610212002).

\section{REFERENCES}

[1] Zhen Liu. Research on the Intelligent Built in Test Fault Diagnosis Method and Its Applications to More-Electric Aircraft Electrical Power System. Xian: Northwestern Polytechnical University, 2006.

[2] Liu Yong, Li Wenhai, Chen Yuliang. Research on intelligent Fault Diagnosis Based on Artificial immune System, Computer Measurement \& Control, Vol. 18, 2010, pp. 2694-2723.

[3] LIU Xin-min, WEN Xi-sen, QIU Jing, et al. False Alarm Filter of Mechatronics Built2in Test Based on the Hidden Markov Model, ACTA ARMAMENTARII, Vol. 26, 2005, pp. 387-389.

[4] Yong Deng ; Yibing Shi ; Wei Zhang. An Approach to Locate Parametric Faults in Nonlinear Analog Circuits, IEEE Transactions on Instrumentation and Measurement, Vol. 61, 2012, pp. 2200-2211.

[5] GAO Yingjie, KONG Xiangdong, ZHANG Qin. Wavelet Packets Analysis Based Method for Hydraulic Pump Condition Monitoring. Journal of Mechanical Engineering, Vol. 45, 2009, pp. 80-88.

[6] BARUAH P, CHINNAM R B. HMMs for diagnostics and prognostics in machining process. International Journal of Production Research, Vol. 43, 2005, pp. 358-367.

[7] Jianbo Yu. Health Condition Monitoring of Machines Based on Hidden Markov Model and Contribution Analysis. IEEE Transactions on Instrumentation and Measurement, Vol. 61, 2012, pp. 2200-2211.

[8] ZHINONG LI, JIE HAN, JUNJIE SUN. Fault Recognition Method Based on Independent Component Analysis and Hidden Markov Model, Journal of Vibration and Control, Vol. 13, 2007, pp. 125-137.

[9] Lijia Xu, Jianguo Huang, Houjun Wang, et al. Bing Long. A Novel Method for the Diagnosis of the Incipient Faults in Analog Circuits Based on LDA and HMM, Circuits Systems and Signal Processing, Vol. 29, 2010, pp. 577-600.

[10] Lijia Xu, Study on Fault Prognostic and Health Management for Electronic System. Chengdu: University of Electronic Science and Technology of China. 2009.

[11] Song Liwei. Analog circuit fault diagnosis based on Wavelet Analysis and Neural Network. Changsha: Hunan University. 2012.

[12] Xu Lijia, Huang Jianguo, Wang Houjun. A Method for the Diagnosis of the Incipient Faults in Analog Circuits Using HMM. Journal of Computer-Aided Design \& Computer Graphics, Vol. 22, 2010, pp. 1215-1222.

[13] WANG Guo-feng, LI Yu-bo, QIN Xu-da, et al. Fault Diagnosis of Rolling Bearing Based on TVAR and HMM, Journal of Tianjin University, Vol. 43, 2010, pp. 168-173. 